\title{
Nutzung von CAD-Funktionalität zur Erzeugung des Einguß- kanales auf Modellplatten für DISAMATIC-Formanlagen
}

\author{
Norbert Miersch
}

\section{Einleitung}

Die vorhandenen Funktionalitäten im 3D-CAD-System SolidWorks 99, wie die nutzerspezifische Erstellung und Verwendung von Bibliotheksfeatures, können für eine rechnerunterstuitzte Modellgenerierung genutzt werden. Im folgenden Artikel soll der Ablauf für die Generierung des Eingußkanales mittels Bibliotheksfeatures im CAD-System SolidWorks 99 dargestellt werden.

Der Eingußkanal ist ein Teil des Anschnitt- und Speisersystems, siehe Abbildung 1. Die Gestaltung des Modelles einschließlich seinem Anschnitt- und Speisersystem basiert auf der Festlegung des Rohteiles und seiner Kriterien.

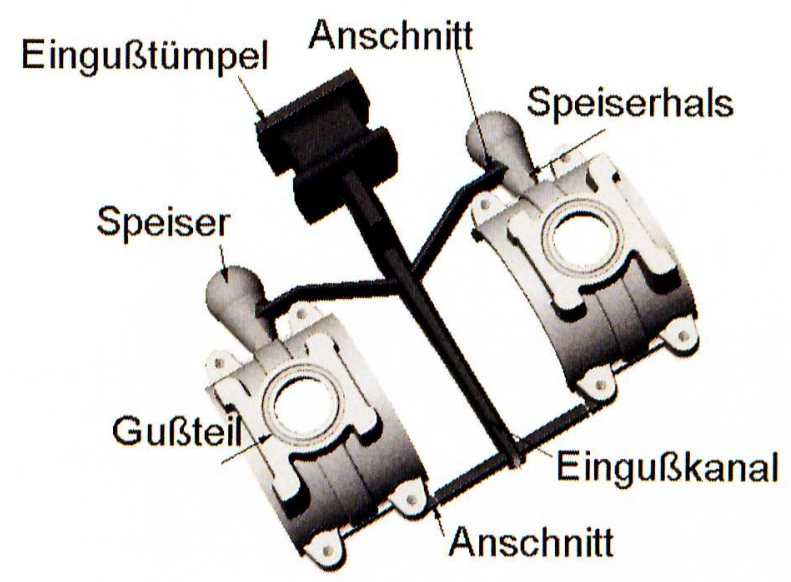

Abbildung 1: Gußteil mit Anschnitt- und Speisersystem für eine DISAMATICFormanlage

Die wichtigsten Rohteilkriterien sind:

- Teilung

- Kerne, Gestalt und Abmessungen

- Vorgießbare Formelemente, Gestalt und Abmessungen.

- Bearbeitungszugaben

- Aushebeschrägen

- Gußradien

- Genauigkeitsklasse

- Technologisch notwendige Formelemente, wie Rippen, Verbindungsstege etc.

- Angaben zur fertigungsgerechten Gestaltung

- Anschnitt- und Speiserlage

- Spannpunkte, Auflagestellen

- Kennzeichnung

- Stoffeigenschaften nach der Wärmebehandlung

- Korrosionsschutz

Auf der Grundlage einer Modellzeichnung wird eine Modellplatte mit dem Modellaufbau gefertigt. Die Mo- dellzeichnung stellt dabei die Ergänzung der Rohteilzeichnung um gießtechnologische Angaben dar. Die Rohteilzeichnung schließlich verkörpert das Gußstück nach der Entnahme aus der Form und dem Putzen, ohne Kernmarken und ohne Anschnitt- und Speisersystem. [2]

Die wichtigsten Merkmale einer Modellzeichnung bezogen auf das

DISAMATIC-Formverfahren sind:

- Modellteilung, Abballungen

- Kernmarkengestalt, Kernmarkenabmessungen

- Abmessungen und Gestalt von Anschnitten, Läufen und Speisern

- Güiteklasse

- Modellwerkstoff

- Schwindmaße

- Kennzeichnung von Modell

- Abmessungen der Kerneinlegermaske, Werkstoff der Kerneinlegermaske

- Kernmarkenabmessungen in der Kerneinlegermaske

- Kernkastenteilung, Kernkastenwerkstoff, Kennzeichnung der Kernkästen

\section{Grundlegendes zur CAD-Technik}

Moderne CAD-Systeme sind 3D-Hybridmodellierer, d. h. es können Draht,- Flächen,- und Solidmodelle erstellt und verändert werden. Das CAD-System CATIA V4 besitzt diese Eigenschaften. Die Arbeit mit Volumenmodellen hat in den letzten Jahren mehr an Bedeutung gewonnen, da hier Regelgeometrien komfortabel und transparent erzeugt werden können. Über eine Konstruktionslogik (Parteditor, Featuremanager) kann in die Struktur des erzeugten Werkstuickes oder der Baugruppe problemlos eingegriffen werden.

Volumenmodelle genügen den Anspruch einer geometrisch vollständigen und eindeutigen Darstellung realer, idealisierter oder erdachter Objekte. Sie bilden zu jedem Zeitpunkt das Volumen der Objekte vollständig ab. [6]

Werkstuicke können auch durch Freiformflächen begrenzt sein. Freiformflächen und Werkstücke aus Freiformflächen können aber auch durch das Aufnehmen von Meßpunkten mit einer Meßmaschine erzeugt werden (digitalisieren). Eine Punktewolke wird in eine Flächendefinition überführt. Je nach Strategie der Meßpunktaufnahme ist das in der Regel mit einem sehr hohem manuellen Arbeitsaufwand verbunden.

Freiformflächen versucht man möglichst unter Beachtung einer Elementhierarchie auf ein Volumenmodell zurüickzufuihren. 
Ein Werkstiuck kann aber auch aus Flächen- und Volumenstrukturen bestehen. Das ist vor allem dann notwendig, wenn aus den Flächeninformationen kein geschlossenes Volumen erzeugt werden kann. In CATIA V4.2 ist es bereits möglich, die Volumen- und Flächenstruktur (Konstruktionslogik) in einem Strukturmanager abzubilden. Flächen- und Drahtinformationen sind vor allem für eine NC-Bearbeitung nutzbar.

3D-Volumenmodelle haben folgende Vorteile gegenuiber Flächenmodellen:

1. Auswertung von Volumen oder Teilvolumen durch Analysefunktionen. Berechnung der Volumenoberflächen, Massenmittelpunkte, Hauptträgheitsachsen, Trägheitsmomente, Volumenberechnungen, abgeleitete Berechnungen wie Masseberechnungen etc.

2. Möglichkeit der Anwendung von Boolschen Operationen. Erzeugen von Schnitten in Volumen (Subtraktion), Austragen von Volumen (Addieren), Vereinigen von Volumen (Union), Bilden von Schnittmengen. Feststellen von Durchdringungstiefen durch Anwendung von Schnittoperationen.

3. Anwendung einer 3D-Featuretechnologie, die auf Boolschen Operationen beruht. Features sind z. B. Bohrungen, Nuten, Rippen, Auszugsschrägen, Paßfedern, Taschen aber auch Normteile.

4. Komplexe Nutzung der 3D-Parametrik. Erzeugung von Abhängigkeiten zwischen geometrischen Größen. Verwendung von Gleichungen. Anwendung der Abhängigkeiten bei der Generierung von Variantenkonstruktionen. Diese Funktion ist nicht bei allen Volumenmodellierern implementiert. (z. B. nicht bei HP-SolidDesigner)

5. Plausibilitäts- und Interferenzkontrollen bei Baugruppenkonstruktionen.

SolidWorks ist ein 3D-Volumenmodellierer und besitzt diese oben genannten Eigenschaften. Er wird deshalb als ein komfortables und leistungsfähiges Werkzeug für die Featuregenerierung angesehen.

\section{Generierung von Gießläufen mittels SolidWorks 99}

\subsection{Theoretische Grundlagen}

Die Berechnung von Anschnittsystemen für DISAMATICFormanlagen erfolgt auf der Grundlage eines druckgesteuerten Anschnittsystems [1];[4]. Den maßgeblichen Faktor für die Gießleistung bildet die metallostatische Druckhöhe. Um gleiche Durchflußmengen in unterschiedlichen Druckhöhen zu erreichen, müssen die Anschnittsquerschnitte in Abhängigkeit von der Druckhöhe berechnet werden.

\section{Unterschiedliche Druckhöhen entstehen:}

- Wenn die Plattenbelegung mehrere vertikal angeordnete Modelle erfordert.

- Wenn durch die geometrischen Abmessungen die Modellplatte in vertikaler Höhe mit nur einem Modell belegt werden kann. Um eine gleichmäßige
Formfuillung zu erreichen, werden in der Regel für die oberen Anschnitte $75 \%$ und für die unteren Anschnitte $25 \%$ des Gießgewichtes zu Grunde gelegt.

- Wenn ein druckentlastetes Anschnittssystem verwendet werden soll.

Die Grundlage der allgemeinen Anschnittsgleichung bildet die Gleichung nach Torricelli als ein Sonderfall der Bernoullischen Gleichung. Sie wird korregiert mit einem empirischen Wert m, der die Reibungsverluste berücksichtigt.

$v=m \cdot \sqrt{2 \cdot g \cdot H}$

Unter Berücksichtigung des Volumenstromes bei stationärer Strömung (Kontinuitätsgesetz) und der Bestimmung der in einer Zeiteinheit durch den Gießlauf fließenden Flüssigkeitsmenge ergibt sich:

$\frac{G}{t}=\theta \cdot A \cdot m \cdot \sqrt{2 \cdot g \cdot H}$

Die Anschnitte können dann zu

$A=\frac{G}{\theta \cdot t \cdot m \cdot \sqrt{2 \cdot g \cdot H}}$

und

$A=\frac{G}{\theta \cdot t \cdot m \cdot \sqrt{2 \cdot g \cdot H_{m}}}$

berechnet werden. Dabei bedeuten:

$\mathrm{v}$ : Strömungsgeschwindigkeit ................... $\mathrm{m} / \mathrm{s}$

$\mathrm{g}$ : Erdbeschleunigung ................................. $\mathrm{m} / \mathrm{s}^{2}$

$\mathrm{H}$ : Druckhöhe ................................................... mm

$\mathrm{H}_{\mathrm{m}}$ : Mittlere Druckhöhe für druckent-

lastetes Anschnittssystem .........................mm

t : Gießzeit .................................................... min

$\mathrm{m}:$ Geschwindigkeitsfaktor

$\theta$ : Dichte des flüssigen Metalles .................. $\mathrm{g} / \mathrm{cm}^{3}$

A : Anschnittsquerschnitt ................................ $\mathrm{mm}^{2}$

a : Seitenlänge der kürzesten Seite des

Trapezquerschnittes $\mathrm{mm}$

$\mathrm{G}$ : Gießgewicht ........................................ Kg

Für die Gestaltung der vertikalen Gießläufe haben sich im Zusammenhang mit einer zerstörungsfreien Entformung trapezförmige Querschnittsformen bewährt [1]. Die Dimensionierung des vertikalen Gießkanales kann nach drei Methoden bestimmt werden.

\section{Bestimmung des größten Querschnittes und Nutzung eines Schrägungswinkels.}

Bei dieser Methode wird der größte Eingußquerschnitt aus der Summe der Einzelanschnittsquerschnitte errechnet. Die Fläche des größten Eingußkanalquerschnittes sollte 10 - $20 \%$ größer sein als die Summe der mit ihm verbundenen Querschnitte, [1]. Der berechnete Kanalquerschnitt wird dann einem Tabellenwert angepaßt und mit einem Schrägungswinkel (Alpha) versehen. Siehe Abbildung 2. 


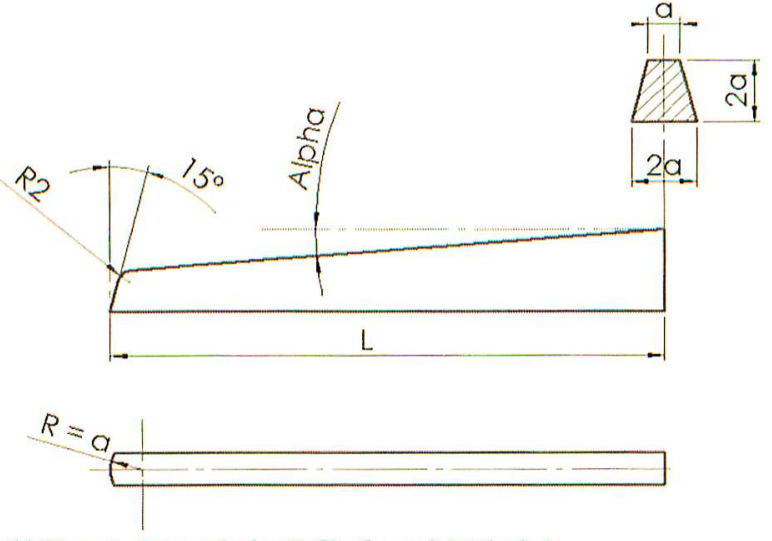

Abbildung 2: Geometrie des Gießlaufes nach Methode 1

2. Berechnen und lineares Verbinden der Eingußquerschnitte in Abhängigkeit von den zu versorgenden Gußstückgewichten.

Eine bessere Methode zur Berechnung des Gießkanales ist die Zusammensetzung aus einzelnen Querschnitten, die nach obengenannter Anschnittsberechnung erfolgt. Bei der Dimensionierung wird das jeweilig zu füllende Gußstiickgewicht unter der entsprechenden ferrostatischen Druckhöhe berücksichtigt. Der Eingußquerschnitt an der entsprechenden Stelle wird ebenfalls 10 - 20\% größer ausgelegt. Bei dieser Methode ist das Volumen des Gießlaufes gegenuiber Methode 1 geringer, siehe Abbildung 3.

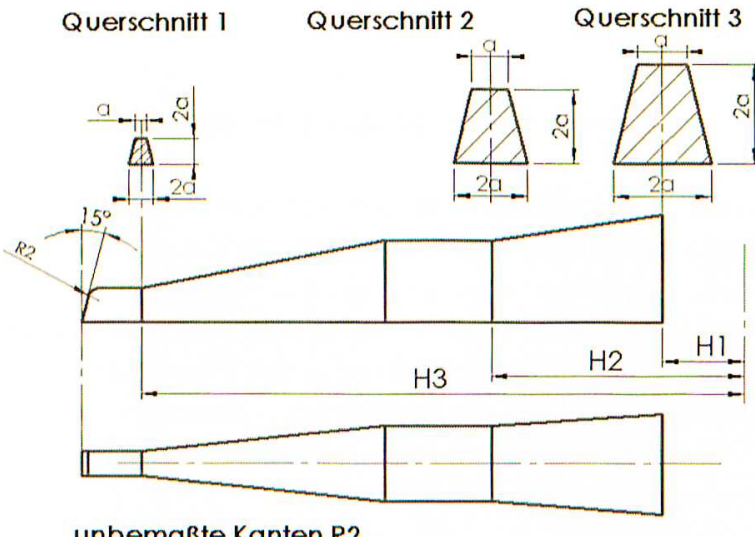

Abbildung 3: Geometrie des Gießlaufes nach Methode 2

Entgegen der in [1] dargestellten Verfahrensweise wird in der Praxis der größte und der kleinste Querschnitt bestimmt und diese Querschnitte linear miteinander verbunden. Das verringert den Aufwand zur Herstellung des Eingußkanales und vermindert durch gleichmäßige Querschnittsänderung mögliche Turbulenzen des einströmenden Metalles. Siehe Abbildung 4.

\section{Berechnung eines druckentlasteten Systems}

Bei dieser Methode wird vor der Berechung des Anschnitts der Querschnitt des Eingußkanales errechnet. Für die Berechnung des Querschnittes im Eingußkanal nach Gleichung 4, liegt hier eine mittlere Druckhöhe zugrunde. Die mittlere Druckhöhe befindet sich im Schwerpunkt des Gußteiles, siehe Abbildung 5. Die Generierung des Eingußkanales kann bei mehreren horizontal übereinanderliegenden Gußstuicken nach Methode zwei, Abbildung 2 durchgefuihrt werden.

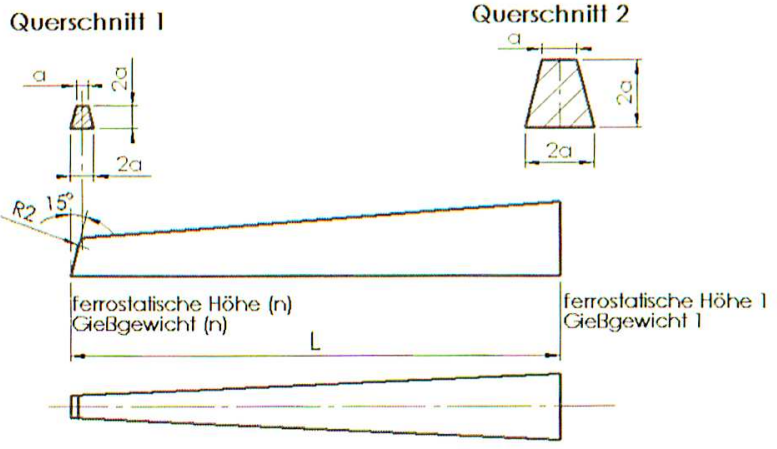

unbemaßte Kanten R2

Abbildung 4: Geometrie des Gießlaufes nach modifizierter Methode 2

\section{druckentlastetes Anschnittsystem}

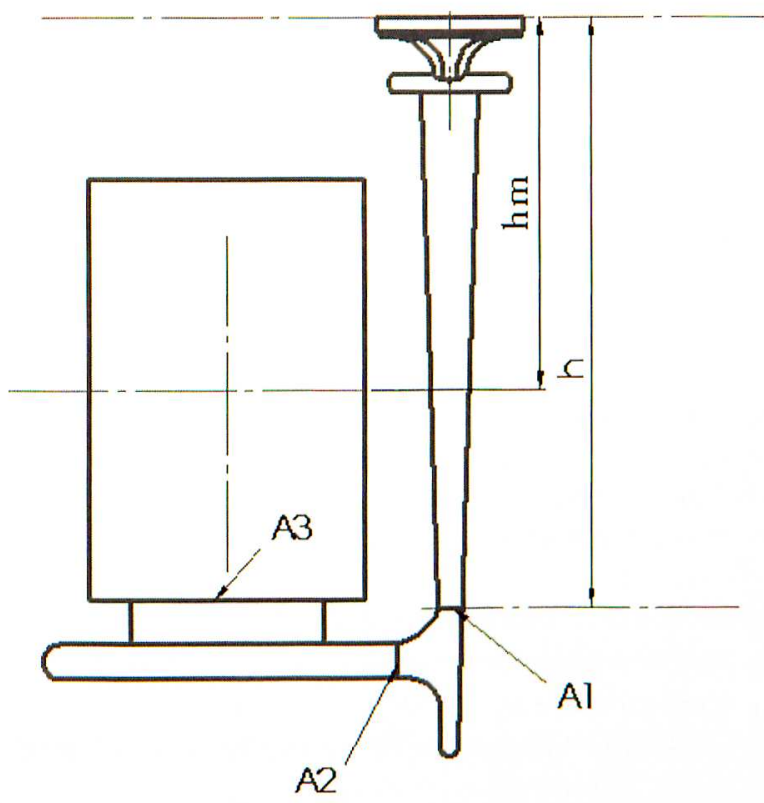

Abbildung 5: Aufbau eines druckentlasteten Anschnittsystems

\subsection{Festlegungen für die Umsetzung der theoretischen Erkenntnisse}

Um in SolidWorks mittels Featuretechnik einen Eingußkanal nach der modifizierten Methode 2 erzeugen zu können, werden im ersten Schritt folgende Vereinbarungen getroffen:

- Der Eingußkanal wird auf eine Modellhälfte montiert. In Abhängigkeit von den Anschnittsbedingungen, die unter anderem abhängig sind von der Geometrie des Teiles, der Formlage, der Lage der Kerne, sowie den Bearbeitungszugaben, werden Flächen mit hoher Abformgenauigkeit und kompliziert zu formenden Konturen auf der Schwenkplattenseite eingeformt. Das Anschnitts- und Speisersystem wird, wenn es die dafür gestellten Forderungen erfüllen kann, möglichst auch auf der Schwenkplattenseite eingeformt.

- Ein horizontaler Gießlauf wird nicht verwendet.

- Aufgrund der besseren Ausformbarkeit des Modelles werden trapezförmige Querschnitte verwendet.

- Der Eingußkanal darf keine scharfen Kanten aufweisen. Alle Kanten sind mit einem Radius R2 zu verrunden. 
- Die kleinste Kantenlänge des Trapezquerschnittes beträgt $\mathrm{a}=5 \mathrm{~mm}\left(\mathrm{~A}=75 \mathrm{~mm}^{2}\right)$, siehe [1].

- Es muß möglich sein, Gießläufe ohne Querschnittsveränderung, Gießläufe mit Querschnittsveränderung und Gießlaufendstuicke zu generieren.

- Aufgrund einer optimalen Plattenbelegung erhalten Gießläufe oft auch eine Krümmung. Diese wird zur Zeit noch nicht berüicksichtigt, ist aber durch die Nutzung von Austragungen entlang eines Austragungsprofiles grundsätzlich möglich.

\subsection{Hinweise zur Verwendung von Bibliotheksfeatures}

SolidWorks bietet die Möglichkeit häufig verwendete Features organisiert abzulegen. Das abgelegte Feature kann ähnlich wie bei Nutzung von Symbolbibliotheken in ein neues Teil eingefuigt und modifiziert werden. Für die Organisation und Funktionsweise von Features gibt es in SolidWorks zwei Featurearten.

1. Bibliotheksfeatures (Basisfeatures, mehrere verpflichtenden Referenzen möglich)

2. Paletten-Features (Basisfeatures, nur eine verpflichtende Referenz möglich)

Die Nutzung einer rechnerunterstiitzten Generierung des Eingußkanales ist nur durch Nutzung von Bibliotheksfeatures möglich, da für das Austragen des Kanales 2 Querschnitte in einem bestimmten Abstand, d. h. zwei verpflichtende Referenzen notwendig sind.

Bibliotheksfeatures haben folgende Merkmale:

1. Zum Erzeugen eines Features muß ein Basisfeature erzeugt werden.

2. Gleichungen können nicht aus der Featurebibliothek in das Teil übertrtagen werden.

3. Organisation der Featureablage im Dateibaum des Betriebssystems. Anlegen einer organisierten Featurebibliothek.

4. Bibliotheksfeatures haben haben einen eigenständigen Dateityp *.SLDLFP.

5. Nach dem Einfuigen des Features in das SolidWorksTeil wird das Feature im Featuremanager mit einer eigenständigen Bezeichnung abgelegt. Es kann jedoch wieder in SolidWorks-Objekte zerlegt werden.

6. Das eingefuigte Feature im Teil hat keine Beziehung zum Feature in der Bibliothek. Die Zerlegung des Features hat keinerlei Einfluß auf diesen Zustand.

\subsection{Erstellung von Bibliotheksfeatures für den Eingußkanal}

Zur Generierung des Eingußkanales ist es möglich verschiedene Features nach Forderung von Abschnitt 3.1 zu erzeugen. Ein Beispiel für die Erzeugung eines Gießlaufendstïckes wird im nachfolgend beschriebenen Programmablauf erläutert.

Hierbei ist $\mathrm{zu}$ beachten:

- Zwischen den Querschnittsgößen des Eingußkanales wurden im Bibliotheksfeature Gleichungen verwen- det, siehe [1]. Die verwendeten Gleichungen können aus dem Bibliotheksfeature in das aktuelle Modell nicht übertragen werden.

- Für das Einfuigen eines Bibliotheksfeatures werden zwei notwendige Referenzen benötigt. Diese Referenzen sind zwei Ebenen. Werden beide Ebenen in die Bibliothek aufgenommen, wird nach dem doppelten Anwählen der gleichen Ebene das Feature mit der Originallänge eingefügt, die durch das Verändern der Featurebemaßung korregiert werden kann. Wird nur eine Ebene in die Bibliothek aufgenommen muß im Modell eine weitere Referenzebene bzw. Fläche vorhanden sein, um den Eingußkanal einfuigen zu können. Beide Möglichkeiten können in der Bibliothek unter verschiedenen Namen abgelegt werden.

- Zwei optionale Referenzen sind notwendig, um den Eingußkanal auf die richtige Höhe und im richtigen Abstand auf die Modellplatte zu legen. Dazu müssen zwei Kanten der Modellplatte ausgewählt werden.

- Das Abspeichern des Bibliotheksfeatures erfolgt unter folgenden Konventionen < name referenzen $>$ SLDLFP Bsp.: KanalEndstueck_2gleicheEbenen-1Kante.SLDLFP

Abbildung 6 zeigt den Programmablauf, wie mittels SolidWorks ein Bibliotheksfeature für ein Eingußkanalendstiick erstellt werden kann. Abbildung 7 stellt die graphische Oberfläche und den Featuremanager aus SolidWorks dar. Im Featuremanager sind die zur Bibliothek hinzugefügten Features zu erkennen.

\subsection{Einfuigen des Eingußkanales auf die Modellplatte}

Um den Eingußkanal uiber ein Bibliotheksfeature generieren zu können, muß bereits eine Modellplatte (Basisfeature) erzeugt worden sein. Abbildung 8 stellt den Programmablauf zum Einfiigen des Eingußkanalendstuickes auf eine Modellplatte dar. Der Eingußkanal muß auf die Oberfläche der Modellplatte eingefuigt werden, d. h. die notwendigen Referenzen (zwei Ebenen) muissen vor dem Einfiigen vorhanden sein.

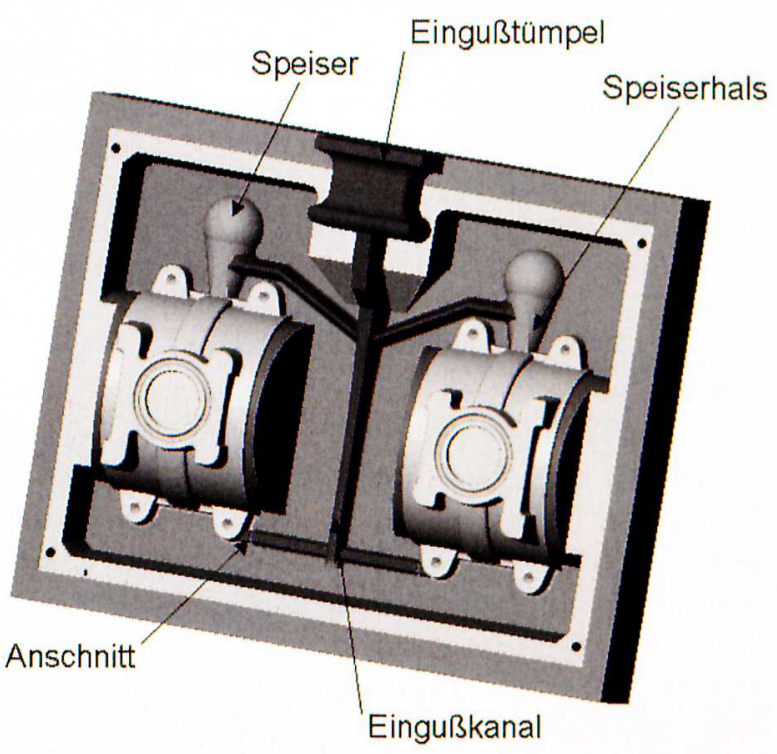

Abbildung 10: Vollständige Modellplatte mit Anschnitts- und Speisersystem 
Er stellung eines Bibliothek sfeatures mit dem CAD-System SolidWorks

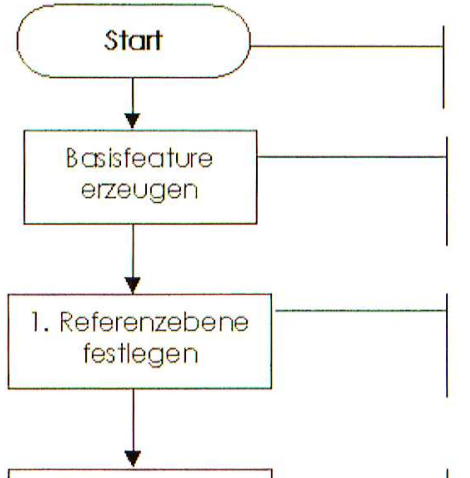

Für die Generierung des Eingusses ist die Erzeugung eines Basisfe atures notwendig Als Basis wurde beim Eirfugen in ein T eil die Modellplatte dienen

Zur Erzeugung des er sten größeren Quer schnittes durch eine Skizze muj eine Referenzebene festgelegt werden. Hierzu wird eine der 3 Standar debenen ausgewahlt.

2. Referenzebene festlegen

\section{Auf 1. Referenzebene}

1. Skizze erzeugen.

Bemaßen der skizze zu einer Kante des

Basisfeatures

Zur Erzeugung des zweiten kleineren Quer schnittes, der der gofiten ferrostatischen $\mathrm{H}$ ohe zuge or dnet wird, muß eine 2 . Ebene erzeugt werden, auf der der 2. Querschnitt skizziert werden $m$ us.

Sizzieren des ersten Querschnittes. Verwendung von Gleichungen für de Abhaingigkeiten der Trapezhöhe und der unteren Trapezs eite. V errunden des Querschnittes mit Radius 2 an den oberen Karten Skizze eindeutig bestimm en

"h1/GGroßer Quer schnitt" = 2*" a1 $10 \mathrm{G}$ roßer Ouer schnitt"

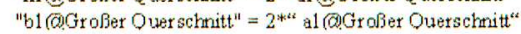

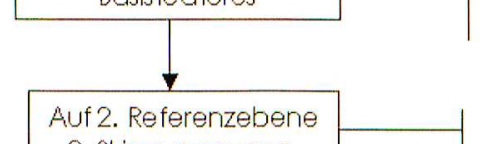

Auf 2. Referenzebene

2. Skize erzeugen.

Kolinearität der

Mittellinie zur 1. Skizze erzeuden.

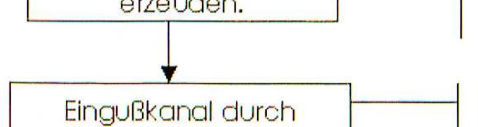

Fingußkand durch beide Skizzen ausformen

chnittebene für

schräges Kanalende erzeugen

Sizzier en des zweiten Quer schnittes. Verwendung von Gleichungen für die A bhängigkeiten der Trapezhohe und der unteren Trapezseite. $V$ errunden des Querschrittes mit Radius 2 an den oberen Kanten.

"h2@Kleiner Querschnitt" = 2*"22@Kleiner Quer schritt" "b2@K1einer Querschnitt"=2*" a2@K1einer Querschnitt"

Einfügen einer Ausformung durch beide Skizzen Dabei mus die Reihenfolge der Ausw ahl beachtet werden.

Weiterhin müssen die gegenüberliegenden $E$ cken dex beiden Trapezseiten angew ählt werden

Diese Ebene kann wher 3 Punkte aus den beiden Skizzen erzeugt werden. Auf dieser Ebene muß die Schrittskizze für das Endstick erzeugt werden

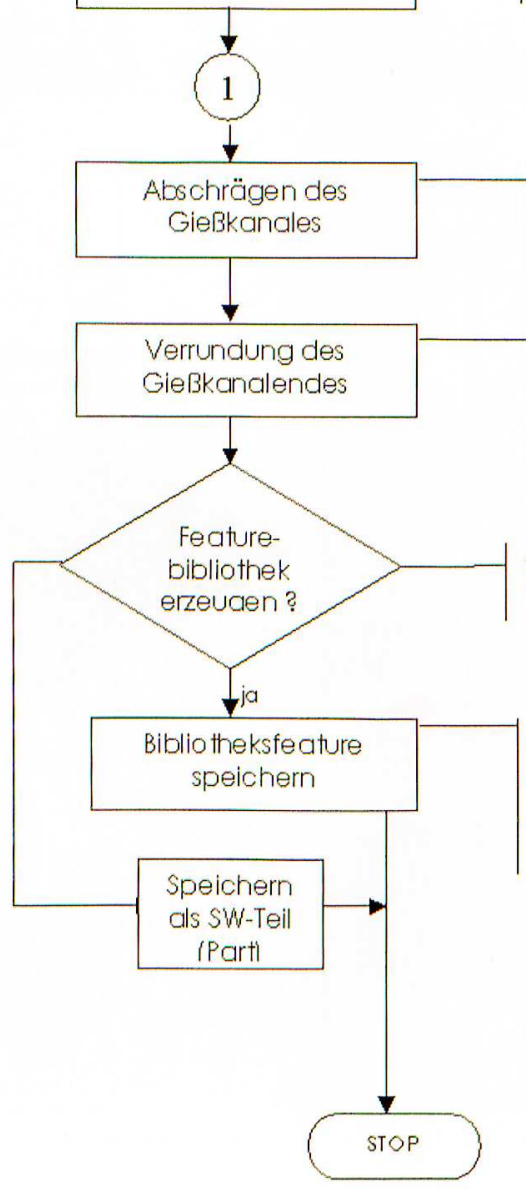

Erzeugung der Abschragung des Gießkanalendes durch das Austragen eines beidseitigen Schrittes auf der zuwor erzeugten Ebere.

Die scharfen Kanten, die durch den Schritt entstanden sind, müssen jetzt mit Radius $\mathrm{R} 2$ vernundet werden.

Soll aus den erstellten Features eine Feaburebibliothek erzeugt werden?

Auswälen der er stellten $\mathrm{F}$ eatures mit $<$ Strg $>$ und linker Maustaste. $<$ Sp eichern unter

KanaIEndstueck 2glEbenenl Kante.SLDLFP

Mit der rechten Maustaste körnen weitere Features zur

Bibliothek hinzugefugt werden 


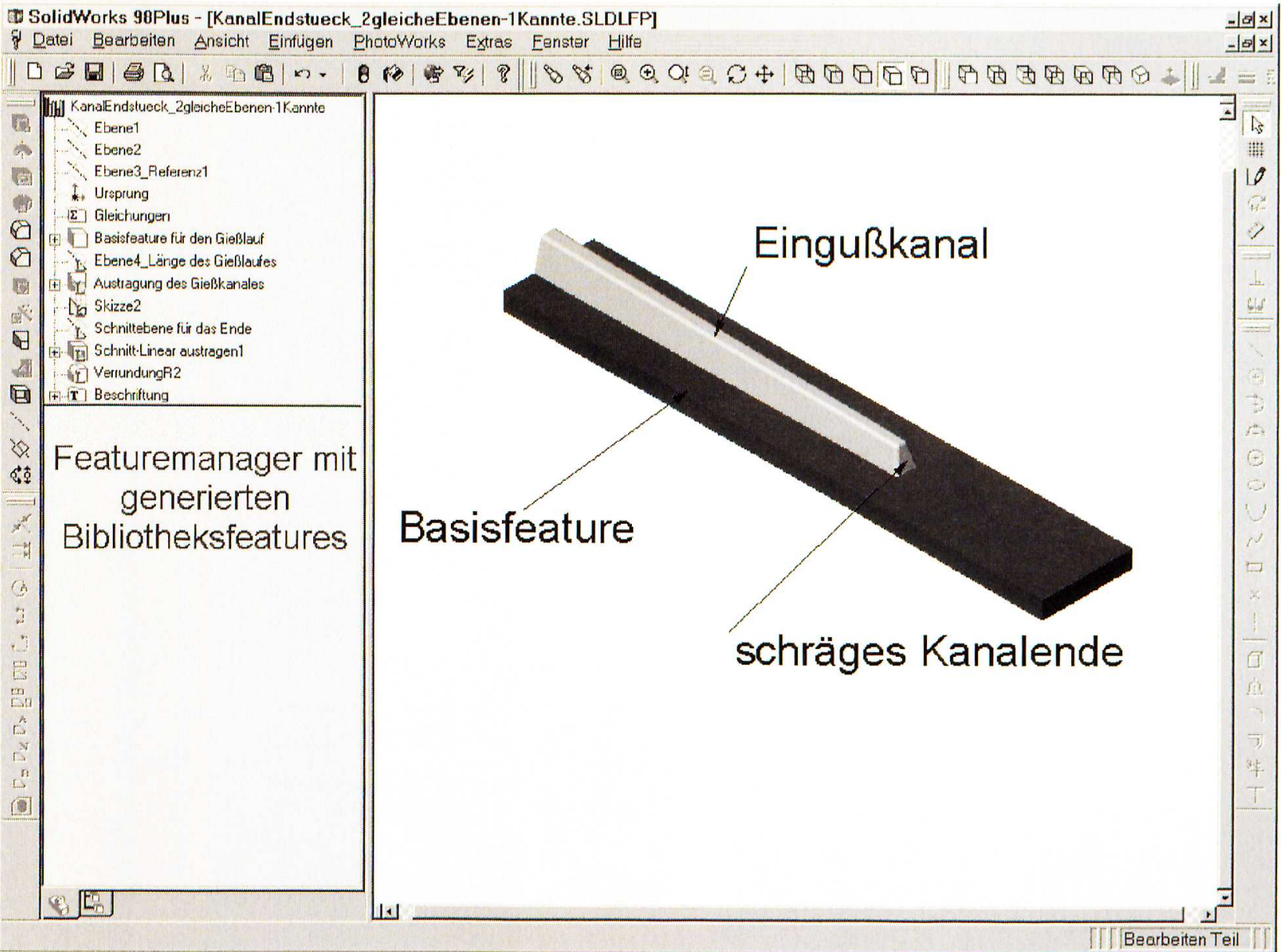

Abbildung 7: Erstellung des Eingußkanalendstückes in SolidWorks.

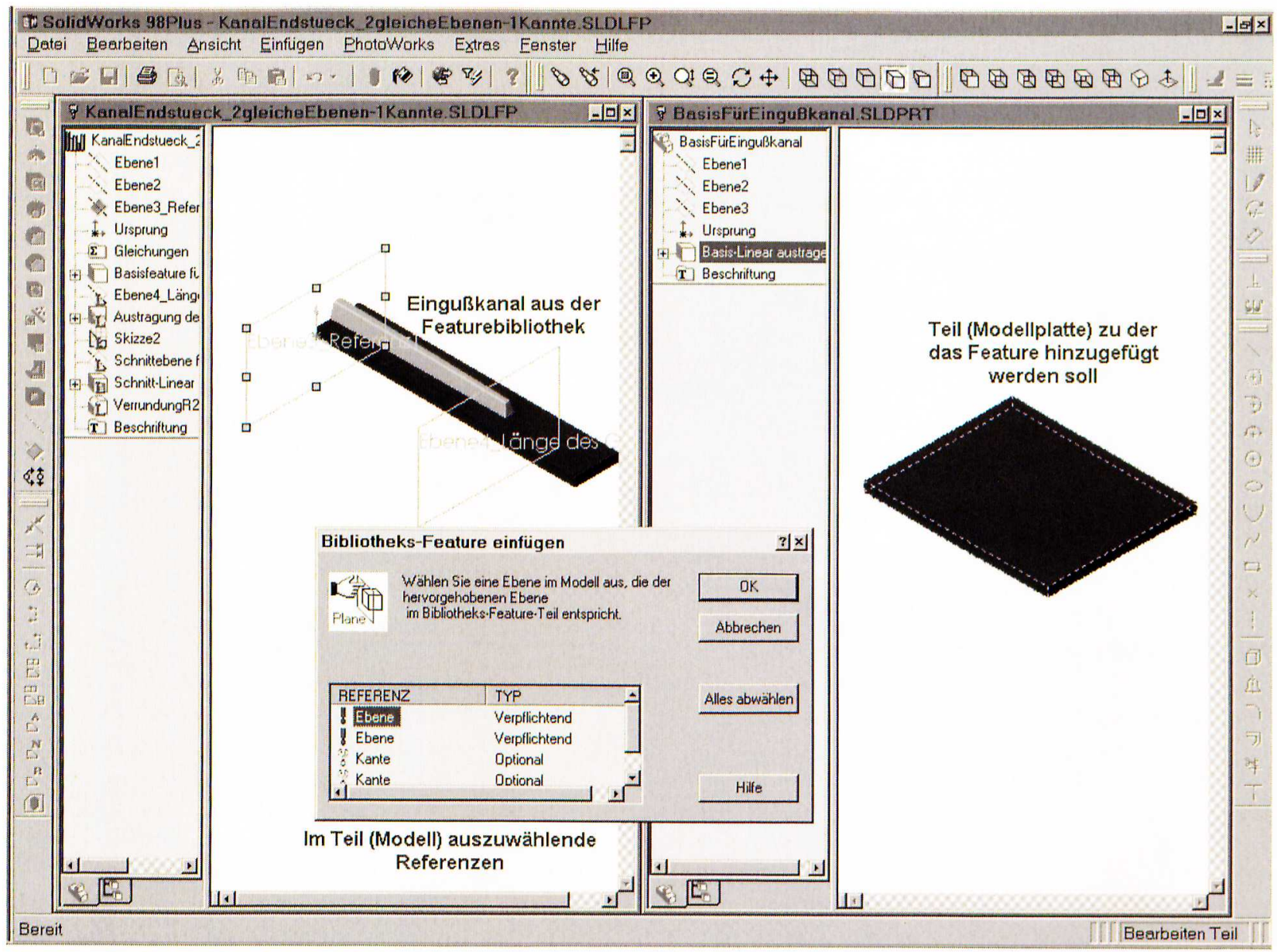

Abbildung 9: Einfügen des Eingußkanales (Bibliotheksfeatures) 


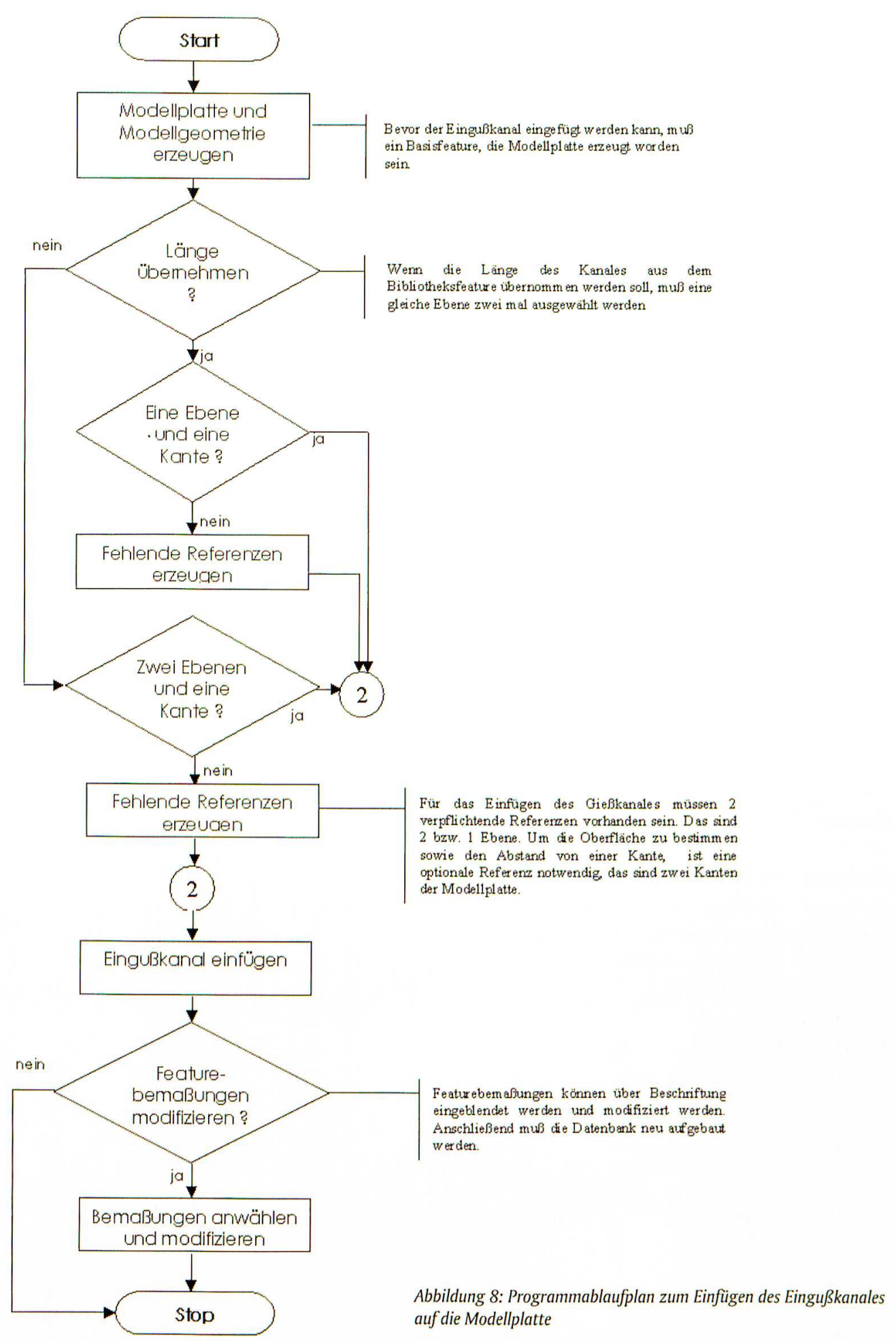

In Abbildung 9 wird im linken Fenster das Bibliotheksfeature und im rechten Fenster das Teil (Modellplatte), sowie die anzuwählenden Referenzen ausgewählt. Abbildung 9 zeigt eine mit Anschnitts- und Speisersystem fertig generierte Modellplatte.

Der Autor dankt an dieser Stelle bei der Gießerei Duktil-Guß Fürstenwalde $\mathrm{GmbH}$ und der Hawle Armaturen $\mathrm{GmbH}$ für die Unterstützung insbesondere für die Bereitstellung von Informationen zur Ausarbeitung des Absatzes 3.1 sowie bei der Analyse der in Abbildung 10 dargestellten Modellplatte.

\section{Zusammenfassung}

Das 3D-CAD-System SolidWorks 99 ist ein leistungsfähiges CAD System, daß neben der Nutzung implementierter Funktionen auch die Möglichkeit bietet, nutzerspezifische Features zu erstellen. Diese Features können organisiert in einer Verzeichnisstruktur abgelegt werden, um sie dann in ein aktuelles Problem einzufuigen zu können. Ein Beispiel für eine mögliche nutzerspezifischen Anwendung zeigt die Generierung eines Eingußkanales auf Modellplatten von DISAMATIC-Formanlagen. Der vorhandene Ablauf kann als Grundlage für die Generierung weiterer Features, auch fuir die Bearbeitung anderer Problemen dienen. 


\section{Literatur}

[1] Georg Fischer Disa GmbH: Handbuch der Anwendungstechnik fur Disamatic 2110 MK2 Sandformsystem. September 1995.

[2] Hofmann, I.; Spangenberg, J.; Miersch, N.; Amobos, E.; Pfisterer, W.: Rechnergestiitzte Generierung des Rohteiles im Rahmen der Angebotsbearbeitung für Neuteile Optimierungsstrategien für Gußstücke. Tagungsband zum Eröffnungskolloquium „Innovative Gießerei“, Teilprojekt „Indirekte Wertschöpfungskette“, Forschungsschwerpunkt „Rationellere Angebotsbearbeitung“. Ottovon-Guericke-Universität Magdeburg. Fakultät für Maschinenbau. Magdeburg, 17. April 1998.

[3] Hogh, J.; Marx, P.; Padmanabhan, S.; Rall, U.; Stöcker, H.; Winkler, H.: Das CAD / CAM / CAE-System Catia Version 4. Der Leitfaden mit praktischen Übungen. 3. Auflage. Verlag moderne Industrie, Landsberg / Lech 1997.

[4] Menden, A.: Giesserei-Modellbau, Handbuch. GiessereiVerlag GmbH. Düsseldorf 1991.

[5] SolidWorks Corporation Concord: SolidWorks 98Plus Benutzerhandbuch. Dokumentnummer SWXUGDEU061599. Massachusetts, 1999.

[6] Spur, G.; Krause, F.-L.: Das virtuelle Produkt. Management der CAD-Technik. Carl Hanser Verlag München Wien, 1997.

\section{Autor}

Dipl.-Ing. Norbert Miersch

Technische Fachhochschule Wildau

Fachbereich Ingenieurwesen /

Wirtschaftsingenieurwesen

Labor für CAD und FEM

Bahnhofstraße

15745 Wildau

Tel. (0 33 75) 508-161

E-Mail: miersch@hrz.tfh-wildau.de 